\title{
Optimizing Patient Positioning During Dermatologic Surgery
}

\author{
Emily Powell, MD; Jacqueline Guidry, MD; Ida Orengo, MD
}

Patient positioning is an important technique for tension control during dermatologic surgery. Increasing tension across a surgical site during excision and decreasing tension during closure ultimately reduces the work required to complete procedures and increases efficiency. These techniques should be considered in areas of high tension and repetitive motion such as the back and lower extremities.

Cutis. 2021;107:43-44.

\section{Practice Gap}

Practical patient positioning is a commonly overlooked method of tension control during excision and repair that allows for easier closure. ${ }^{1}$ Although positioning is a basic step in dermatologic surgery, it often is difficult and awkward for both the patient and physician. Here, we describe basic principles in patient positioning that increase tension across the surgical site during excision and reduce tension during closure. By reducing the amount of work required for excision and closure, procedures are completed more quickly, which increases efficiency. These techniques should be considered during dermatologic surgery at sites that are subject to both high tension and repetitive motion, such as the upper back and lower extremities.

\section{Technique: Upper Back Procedures}

When removing lesions on the upper back, lying completely prone is uncomfortable for the patient and leaves the shoulders hyperextended. ${ }^{2}$ Instead, position the patient with the arms extended anteriorly, hugging a pillow, while lying prone or on one side (Figure 1). In this position, excision of the lesion is facilitated by increased tension across the upper back. In addition, this position is notably more comfortable for the patient. During closure, the patient should lie on the side contralateral to the surgical site, with the elbow resting at the hip and the ipsilateral arm lying parallel to the torso (Figure 2).
Following procedures on the upper back and shoulders, we typically recommend that the patient wear an arm sling on the ipsilateral side for 1 week. Doing so reliably limits mobility postoperatively and does not require the patient to constantly monitor their movement.

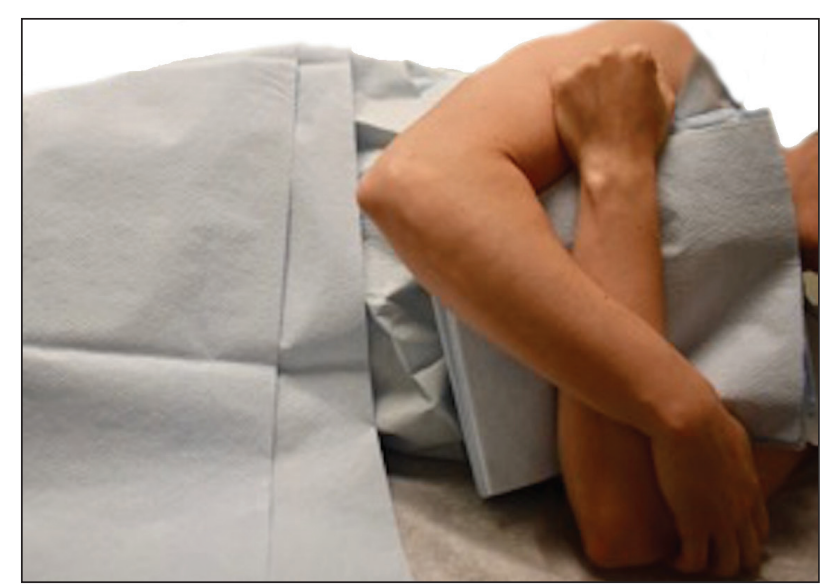

FIGURE 1. Positioning the patient for excision of lesions on the upper back.

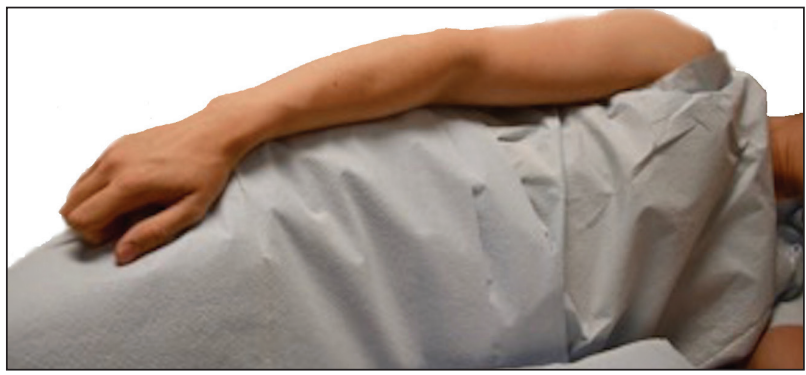

FIGURE 2. Positioning the patient for closure of lesions on the upper back.

Dr. Powell is from the Department of Dermatology, Tulane University, New Orleans, Louisiana. Drs. Guidry and Orengo are from the Department of Dermatology, Baylor College of Medicine, Houston, Texas.

The authors report no conflict of interest.

Correspondence: Emily Powell, MD (epowell4@tulane.edu).

doi:10.12788/cutis.0147 


\section{Technique: Lower Extremity Procedures}

Anterior Lower Extremity-During excision of a lesion on the anterior lower extremity, we recommend that the patient be positioned with their knee bent and heel resting on the examination table. Ideally, the knee is flexed at approximately a $45^{\circ}$ angle (Figure 3 ). ${ }^{3}$ In this position, excision of the lesion is facilitated by increased tension across the anterior lower extremity. During closure of these lesions, the patient should lie supine with the knee fully extended and the leg resting on the surgical bed or a pillow.

Posterior Lower Extremity-During excision of lesions on the posterior lower extremity, the patient should be positioned lying prone, with the knee fully extended, resting on the surgical bed or a pillow, which facilitates excision of the lesion by increasing tension across the site. During closure of these lesions, the patient should lie on the side contralateral to the surgical site, with the leg fully extended for support. The surgical leg should be flexed at the knee at approximately a $45^{\circ}$ angle (Figure 4 ).

\section{Practice Implications}

Despite being an important step, patient positioning is an often-overlooked component of dermatologic surgery. Positioning becomes even more important in areas of high tension and repetitive motion, such as the upper back and lower extremities, where the risk of wound dehiscence and poor scar cosmesis is increased. ${ }^{1}$ Experienced dermatologic surgeons should utilize patient positioning, taking advantage of tension instead of working against it.

We have found that these 2 simple principles can aid in simplifying the excision and repair processes. Increasing tension across the surgical site during excision reduces the work required by the surgeon to reach the appropriate depth. Conversely, decreased tension across the surgical site decreases the work required for closure. These principles should be considered prior to the procedure; the patient should then be positioned in a way that maximizes tension across the surgical site during excision and minimizes tension across the surgical site during closure.

Incorporating these techniques, especially at sites that are subject to both high tension and repetitive motion, such as the upper back and lower extremities, not only increases efficiency but may also reduce the risk for

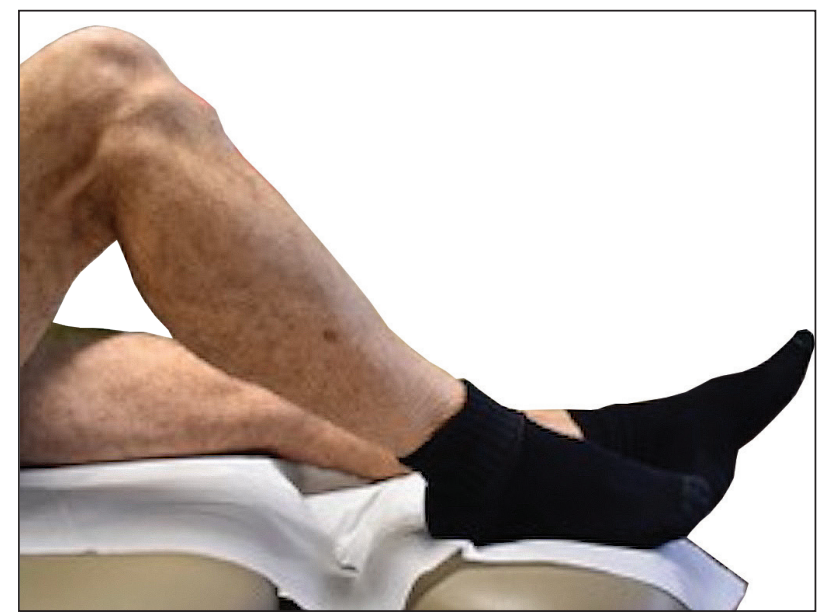

FIGURE 3. Positioning the patient for excision of lesions on the anterior lower extremity.

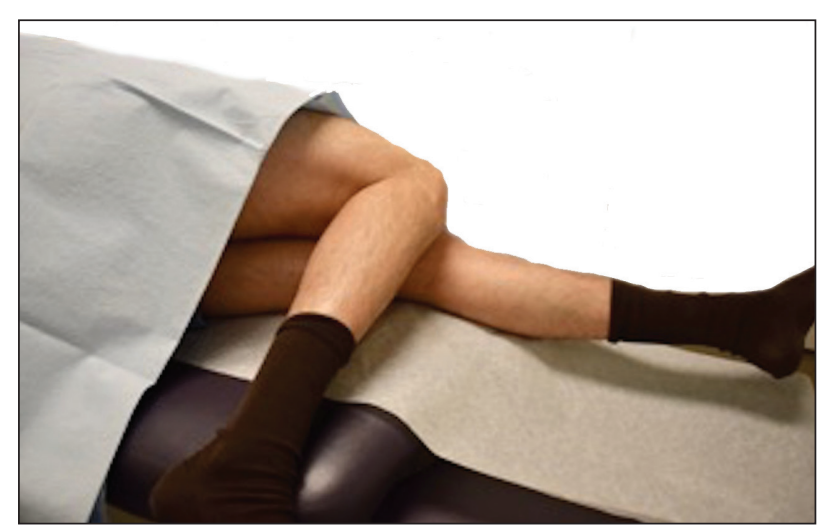

FIGURE 4. Positioning the patient for closure of lesions on the posterior lower extremity.

wound dehiscence once the patient returns home and maintains their normal level of physical activity.

\section{REFERENCES}

1. Rohrer TE, Cook JL, Kaufman AJ. Flaps and Grafts in Dermatologic Surgery. 2nd ed. Elsevier; 2007.

2. Kantor J. Atlas of Suturing Techniques: Approaches to Surgical Wound, Laceration, and Cosmetic Repair. 2nd ed. McGraw-Hill Education; 2016.

3. Kiwanuka E, Cruz AP. Multistep approach for improved aesthetic and functional outcomes for lower extremity wound closure after Mohs micrographic surgery. Dermatol Surg. 2017;43:704-707. 